\title{
BMJ Global Health Chronic kidney disease care models in low- and middle-income countries: a systematic review
}

\author{
John W Stanifer, ${ }^{1,2,3}$ Megan Von Isenburg, ${ }^{4}$ Glenn M Chertow, ${ }^{5}$ Shuchi Anand ${ }^{5}$
}

To cite: Stanifer JW, Von Isenburg M, Chertow GM, et al. Chronic kidney disease care models in low- and middleincome countries: a systematic review. BMJ Glob Health 2018;3:e000728. doi:10.1136/ bmjgh-2018-000728

Handling editor Seye Abimbola

- Additional material is published online only. To view please visit the journal online (http://dx.doi.org/10.1136/ bmjgh-2018-000728).

Received 16 January 2018 Revised 15 February 2018 Accepted 20 February 2018

Check for updates

'Division of Nephrology, Department of Medicine, Duke University, Durham, North Carolina, USA

2Duke Global Health Institute, Duke University, Durham, North Carolina, USA

${ }^{3}$ Duke Clinical Research Institute, Duke University, Durham, North Carolina, USA ${ }^{4}$ Medical Center Library, Duke University School of Medicine, Durham, North Carolina, USA ${ }^{5}$ Division of Nephrology, Stanford University School of Medicine, Stanford, California, USA

Correspondence to Dr Shuchi Anand; sanand2@stanford.edu

\section{ABSTRACT}

Introduction The number of persons with chronic kidney disease (CKD) living in low- and middle-income countries (LMIC) is increasing rapidly; yet systems built to care for them have received little attention. In order to inform the development of scalable CKD care models, we conducted a systematic review to characterise existing CKD care models in LMICs.

Methods We searched PubMed, Embase and WHO Global Health Library databases for published reports of CKD care models from LMICs between January 2000 and 31 October 2017. We used a combination of database-specific medical subject headings and keywords for care models, CKD and LMICs as defined by the World Bank.

Results of 3367 retrieved articles, we reviewed the full text of 104 and identified 17 articles describing 16 programmes from 10 countries for inclusion. National efforts $(n=4)$ focused on the prevention of end-stage renal disease through enhanced screening, public awareness campaigns and education for primary care providers. Of the 12 clinical care models, nine focused on persons with CKD and the remaining on persons at risk for CKD; a majority in the first category implemented a multidisciplinary clinic with allied health professionals or primary care providers (rather than nephrologists) in lead roles. Four clinical care models used a randomised control design allowing for assessment of programme effectiveness, but only one was assessed as having low risk for bias; all four showed significant attenuation of kidney function decline in the intervention arms.

Conclusions Overall, very few rigorous CKD care models have been reported from LMICs. While preliminary data indicate that national efforts or clinical CKD care models bolstering primary care are successful in slowing kidney function decline, limited data on regional causes of CKD to inform national campaigns, and on effectiveness and affordability of local programmes represent important challenges to scalability.

\section{INTRODUCTION}

A majority of the world's population with chronic kidney disease (CKD) lives in lowand middle-income countries (LMIC); yet few data are available to guide the care of persons with CKD in these resource-limited settings, where access to renal replacement therapies is limited. ${ }^{1-3}$ Treatments both

\section{Key questions}

What is already known about this topic?

- Chronic kidney disease (CKD) is a large and growing problem in low- and middle-income countries due to the surging numbers of persons with diabetes, obesity and vascular disease. Understudied early life or environmental exposures are likely substantial contributors.

- Systematic approaches to detecting and managing CKD can substantially mitigate cardiovascular complications and slow progression for end-stage renal disease.

- A recent survey by the International Society of Nephrology indicated that few countries have routine capabilities to screen and diagnose kidney disease, let alone to care for people with end-stage renal disease, thus highlighting the need for innovative programmes to address CKD.

\section{What are the new findings?}

- In our systematic review of existing care models targeting persons with or at risk for CKD, we find that only four countries have described national level strategies to address CKD.

- At a clinical level, while rigorous assessment was rare in the 12 identified programmes, care models supporting primary care providers or allied health workers achieved effectiveness in slowing kidney function decline, while interventions centred on specialty care alone faced challenges.

\section{Recommendations for policy}

- We recommend that national programmes integrate CKD care into chronic disease management programmes, and include measures of potential causes of CKD in surveillance efforts to inform region-specific management strategies.

- We identified programmes supporting allied health workers or primary care providers to take on care of patients with or at risk for CKD as potentially effective in improving outcomes; with further rigorous assessment of affordability and outcomes, these programmes have the highest potential for scalability.

broadly applicable-for example, blood pressure $^{4}$ and glycaemic $\operatorname{control}^{5}$ - and specific-for example, immunomodulation 


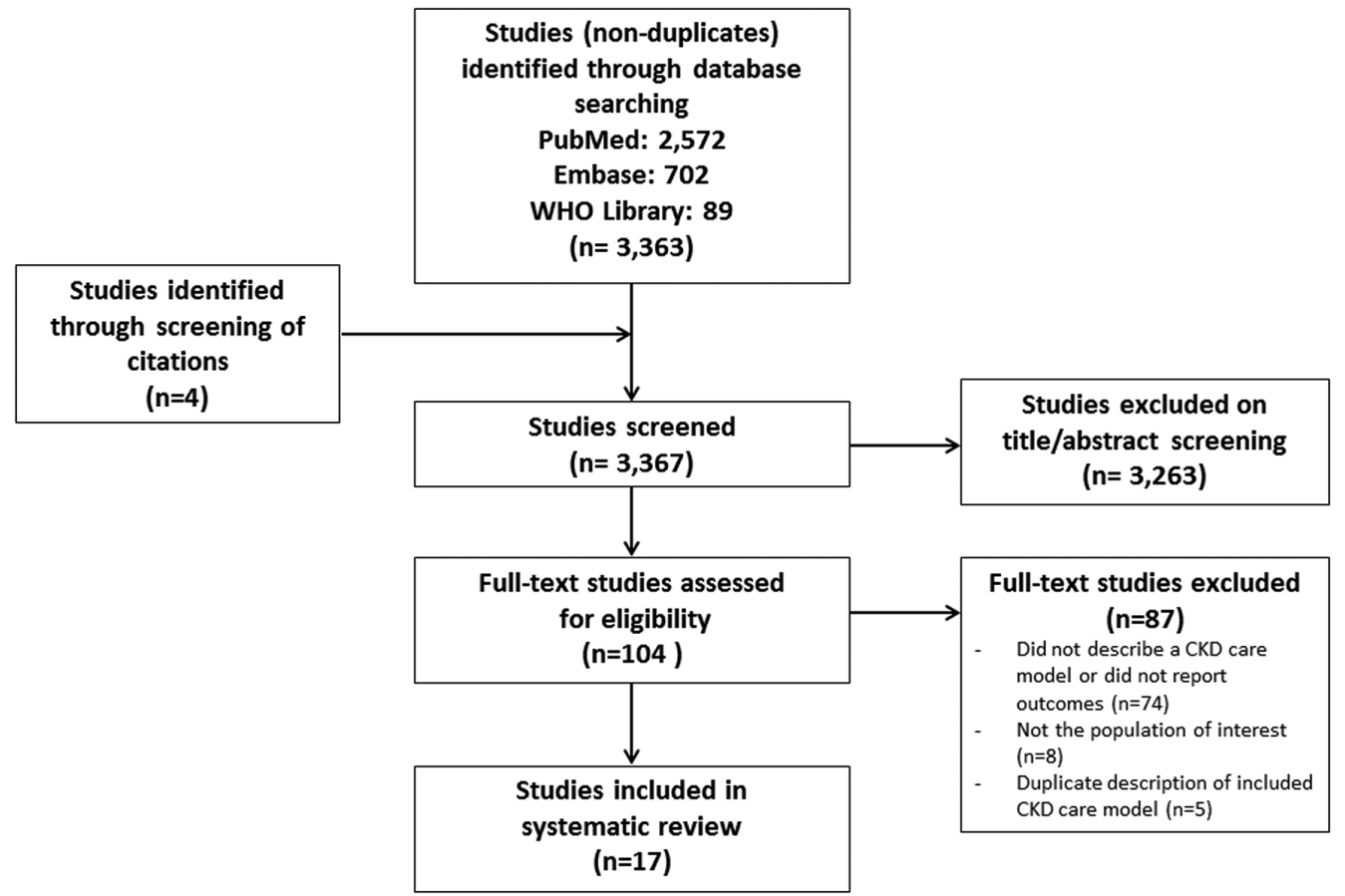

Figure 1 Flow chart showing selection of studies included in review. CKD, chronic kidney disease.

for glomerular disease ${ }^{6}$ or prevention of recurrent kidney stone disease ${ }^{7}$ - can delay progression of CKD. Such interventions take on a special urgency in LMICs since few can afford to provide therapy to the projected numbers of persons who will develop end-stage renal disease (ESRD). ${ }^{12}$ As such, therapies that can prevent or delay progression of CKD are critical.

Chronic disease care models offer a replicable means of providing healthcare services to a specific population. The aims of care models are to improve in service delivery through systematic, measurable changes. ${ }^{8}$ Multidisciplinary CKD care models implemented in high-income countries (HIC) have demonstrated improvements in CKD outcomes, including slowing progression and reducing mortality in the transition to ESRD. ${ }^{9}$ However, in LMICs, implementation of such care similar care models can face substantial challenges, such as scarcity of specialists and other healthcare providers, lack of healthcare systems enabling continuity, and social and financial constraints on the patients.

In order to inform the development of future CKD care models in LMICs, we conducted a systematic literature review to identify and characterise existing care models for adults with non-dialysis requiring CKD living in LMICs. Our goal was to identify salient features of sustainable, scalable CKD care models by evaluating the strengths and limitations of previously implemented models.

\section{METHODS}

\section{Search strategy}

With the assistance of a specialised global health librarian (MVI), we searched the PubMed, Embase and WHO
Global Health Library databases to identify published studies between the years 2000-2017. Search terms included a combination of relevant subject headings and text words for kidney disease (eg, chronic kidney disease, renal insufficiency) and care models or outpatient service provisions (eg, community health services, community health workers, outreach, health promotion) (online supplementary appendix 1). We narrowed our search to only include published studies from LMICs, which we defined according to the World Bank. ${ }^{10}$ We excluded case reports, meta-analyses and editorials. Without limits on language, the search was last generated on 31 October 2017. We supplemented the search by manually adjudicating both the cited and citing references of all included studies.

\section{Selection criteria and definitions}

To be included, studies had to meet three criteria: (1) include adults ( $\geq 18$ years old) residing in an LMIC; (2) include individuals and/or populations with CKD or at risk for CKD in ambulatory or community settings; and (3) describe care models providing healthcare services targeting outcomes relevant to CKD. To qualify for a care model, the study had to outline how healthcare was delivered and how the healthcare needs of the patients and/or population were met. We defined a clinical care model as a programme with the aim of bringing about improvements in service delivery through effecting and measuring change, which distinguished it from standard healthcare delivery. On a national level, healthcare programmes that included a health sector-led sequence of actions or an outline of the way a system or service should function with 
defined outcomes were also considered to meet criteria for being a care model.

\section{Abstraction}

All abstracts were retrieved by two authors (JWS and SA) who then independently reviewed each title and abstract for inclusion. Any disagreement resulted in a joint review of the full article with reconciliation that led to inclusion or exclusion. After review of the abstracts, each article was independently reviewed in full by two authors (JWS and SA). Disagreements regarding the inclusion or exclusion of an article were settled by consensus after joint review, and we estimated inter-rater agreement for inclusion with the kappa statistic. After identifying articles for inclusion, the two authors independently reviewed and abstracted data into a standardised, preformulated form (see tables 1 and 2 for a set of subheadings). The standard data form included authors, year of publication, study design, study site/setting, study size, study population, definition of CKD, study results and characteristics of the care model including target population, mode of healthcare delivery, type of intervention, follow-up period, measured outcomes, costs, uptake, limitations and dissemination efforts. Errors in data extraction were resolved by joint review of the original articles. Study authors for all included studies were contacted for clarifications or requests for additional follow-up data since time of initial publication.

We assessed each included study using standard quality assessment scales for randomised controlled trials or observational studies. ${ }^{11}{ }^{12}$ For case-control and cohort studies, we assessed for quality on the basis of patient selection, comparability and outcome measures. ${ }^{12}$ Randomised controlled trials were assessed by judging the risk of bias using the Cochrane Collaboration's tool for assessing risk of bias. ${ }^{11}$ We categorised studies as low, medium or high on the basis of these criteria.

\section{RESULTS}

We identified 3367 articles, of which 17 were included (figure 1). Inter-rater agreement for inclusion was excellent $(\kappa>0.90)$. The most common reasons for exclusion were studies that did not describe a care model specific to CKD or did not report outcomes of the implemented care model $(\mathrm{n}=74)$, the study examined populations not of interest to our study $(n=8)$ (eg, paediatric populations, high-income settings), or the article was a duplicate description of an included CKD care model $(n=5)$. The 17 articles, published between 2003 and 2017, reported 16 care models from 10 countries, seven of which were classified as upper middle income, two as lower middle income and one as low income (figure 2). Of the 16 care models, we classified four as national level (table 1) and 12 as regional or local-level CKD care models (table 2A,B).

Studies reporting care models at the national level were all classified as low quality on the basis of no quantification of outcomes and limited follow-up. Among studies
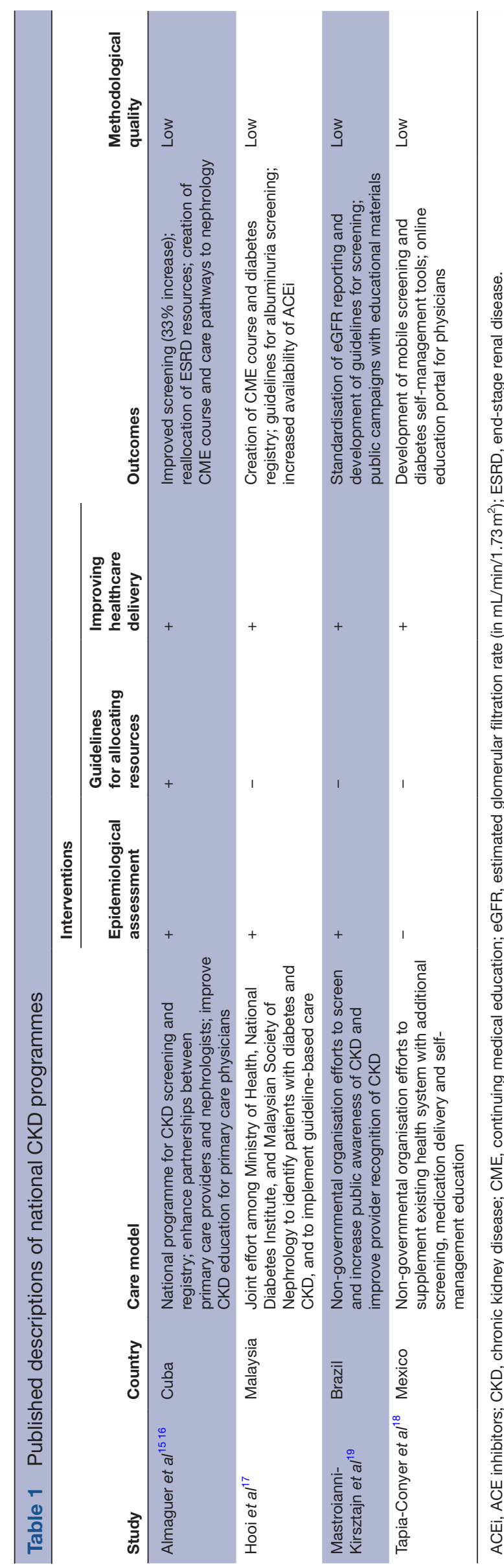


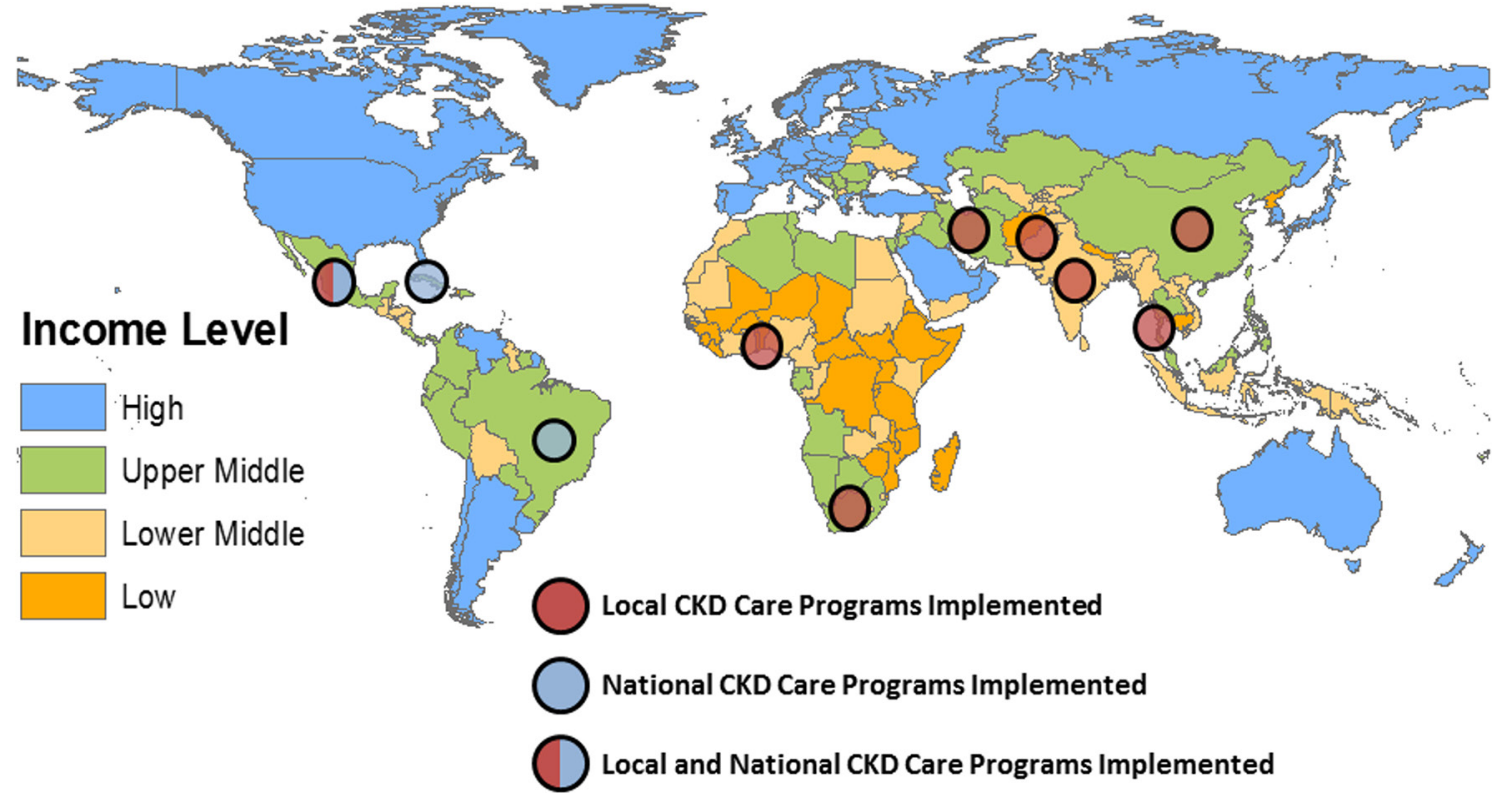

Figure 2 Geographic location of described programmes. CKD, chronic kidney disease.

describing clinical care models with random patient allocation, only one ${ }^{13}$ met criteria for low risk of bias, and among studies at the regional or local level without random patient allocation, only one $\mathrm{met}^{14}$ criteria for medium quality and none met criteria for high quality.

\section{National care models}

Cuba, Malaysia, Mexico and Brazil described national care models (table 1). Two (Cuba and Malaysia) were sponsored in some capacity by Ministries of Health ${ }^{15-17}$; and one-describing a care model in Mexico ${ }^{18}$-was sponsored by a large non-governmental organisation. Cuba's programme was described in two publications: one focusing on nephrology services and continuing medical education activities, ${ }^{15}$ whereas a second the linkages established between nephrologists and primary care providers. ${ }^{16}$ Brazil's Society of Nephrology led its national level efforts. ${ }^{19}$ All four countries' care models focused on enhancing screening, increasing public awareness and improving CKD education for primary care providers. For example, in Cuba, Almaguer et al ${ }^{15}{ }^{16}$ described epidemiology assessments of the CKD burden and causes of kidney disease-related deaths, and use of these data to restructure nephrology services to include a national population-level 'nephrology net'. ${ }^{15}$

To improve primary care provider (PCP) knowledge and recognition of CKD, all four care models reported the development of continuing medical education courses or educational materials, including an online educational portal. ${ }^{18}$ Additional efforts in Cuba and Malaysia facilitated continuity of care with the creation of a CKD registry ${ }^{1516}$ and with the use of a patient-specific diabetes data card to track diabetes-related complications. ${ }^{17}$ In Mexico, there was an emphasis on self-management with development of mobile health (mHealth) technology to enhance medication adherence. ${ }^{18}$

\section{Clinical care models}

Four of the 12 clinical care models undertook assessments of their programme using a randomised control design $^{1320-22}$ (table 2A). Of these, three were focused on patients with CKD, and one of persons at risk for CKD. In the cluster randomised controlled trial described by Jafar $e t a l^{13}$, lay health workers performed family counselling and follow-up with persons with hypertension, and primary care providers received specialised, intensive training in the management of hypertension, resulting in an attenuation in decline estimated glomerular filtration rate (eGFR) in the treatment groups compared with standard care. Two studies reported formation of multidisciplinary clinics to improve quality of care provided to patients with CKD (Mexico and Thailand), and both showed better preservation of eGFR in the treatment versus control groups. Notably in both of these programmes, community health workers or primary care providers (rather than nephrologists) took on the leading roles.

Of the eight clinical care models ${ }^{142-29}$ employing a before-after or prospective comparison to a (non-randomised) usual care group, two focused on the at-risk population (table 2B). In rural India, $\mathrm{Mani}^{27}$ employed local community lay health workers, and in primary care clinic in South Africa, Katz et $a l^{23}$ focused on leveraging existing clinical infrastructure to prevent CKD through enhanced screening for diabetes and hypertension. Both also created context-specific protocols for management of these conditions and improved medication delivery. In the remaining studies, a majority $(\mathrm{n}=4)^{1422252829}$ took a multidisciplinary approach centred around clinic visits, with the largest study by Garcia-Garcia et $a l^{14}$ reporting better preservation in eGFR in the multidisciplinary care patients compared with existing literature. One clinic 


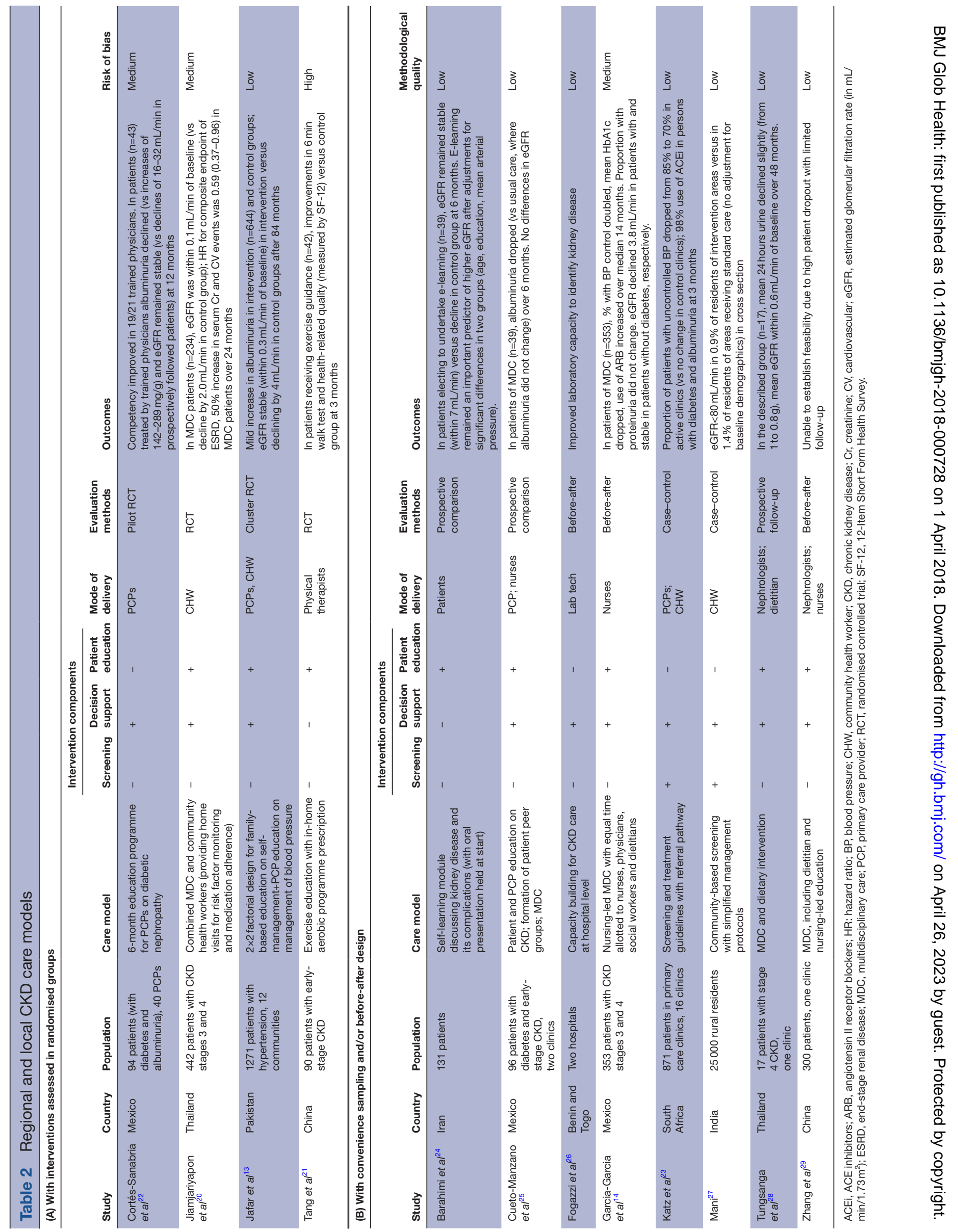


in China straightforwardly described high staff turnover and fractured patient follow-up. None of the clinical care models reported outcomes related to ESRD or cardiovascular events.

\section{Follow-up challenges}

We obtained follow-up data for four of the 16 care models. In Malaysia, recent data suggest the rate of ESRD may be slowing, ${ }^{30}$ but it is unclear whether this trend will continue since estimates for prevalence of CKD remain high. ${ }^{31}$ In Benin and Togo,${ }^{26}$ in partnership with the International Society of Nephrology, educational efforts for providers are ongoing through seminar courses, and a laboratory technician has been trained in urine microscopy; however, financial constraints have prohibited expansion and rigorous outcome assessments. In Mexico, the multidisciplinary CKD clinic organised by Garcia-Garcia et $a l^{14}$ is now in its eighth year, caring for nearly 400 established patients with moderate to advanced CKD. More patients are starting dialysis non-emergently, and four patients have obtained a pre-emptive kidney transplant. For those unable to obtain dialysis or who choose not to, a palliative care programme is now being implemented. Although more patients have insurance, obtaining medications and lab supplies still remains challenging. In India, the services provided by Mani's ${ }^{27}$ care model have been extended into adjacent regions, although governmental support has not been provided for regional or national expansion.

\section{DISCUSSION}

Despite the growing burden of CKD in LMICs, we found few care models have been implemented on a national or local, clinical level to specifically prevent or manage the complications related to CKD. Among the 16 reported care models, we identified several strengths, such as integration of CKD care in national programmes addressing diabetes and a focus on empowering primary care and allied healthcare workers in clinical programmes.
Three care models implemented at the clinical level are ongoing, indicating that innovative and context-specific programmes have the potential for sustainability. At the same time, we identified many challenges and opportunities for implementing systems for CKD care (figure 3). On a national level there is a need for a better understanding of the epidemiology of CKD, and on a clinical level, there is a need for rigorous evaluation of patient outcomes and cost as well as for integration into community and/or national efforts.

The four countries which reported national-level efforts ${ }^{15-19}$ to address CKD are at the forefront of recognising its heavy morbidity and mortality, especially the impoverishing effect of ESRD. As the recent survey assessing global kidney healthcare resources reported, ${ }^{3}$ fewer than one in four surveyed countries had facilities available for routine measurements of serum creatinine or proteinuria, and a vast majority are far from any national approaches to address the CKD burden. Two national-level care models ${ }^{17} 18$ integrated their efforts within a larger strategy to tackle chronic disease; for example, in the case of Malaysia ${ }^{17}$ efforts were coled by an organisation tasked with diabetes management. Current data indicate most persons with CKD have coexisting conditions such as diabetes, vascular disease or obesity, ${ }^{32}$ and an integrated strategy for screening and comanagement may be efficient and effective.

Nonetheless, although national strategies designed to integrate CKD care with other common comorbid chronic conditions such as diabetes and hypertension may be optimal for some settings, in many LMICs, causes of CKD are more heterogeneous than in HICs (Anand et al, unpublished data, 2017). ${ }^{33}$ Only one of the national efforts (Cuba) ${ }^{15} 16$ attempted to understand the major region-specific causes of CKD-a cornerstone to devising national strategies for detection, education and prevention. Japan, which has among the world's highest incidence of IgA nephropathy, developed a urine dipstick-based screening programme in children. ${ }^{34}$

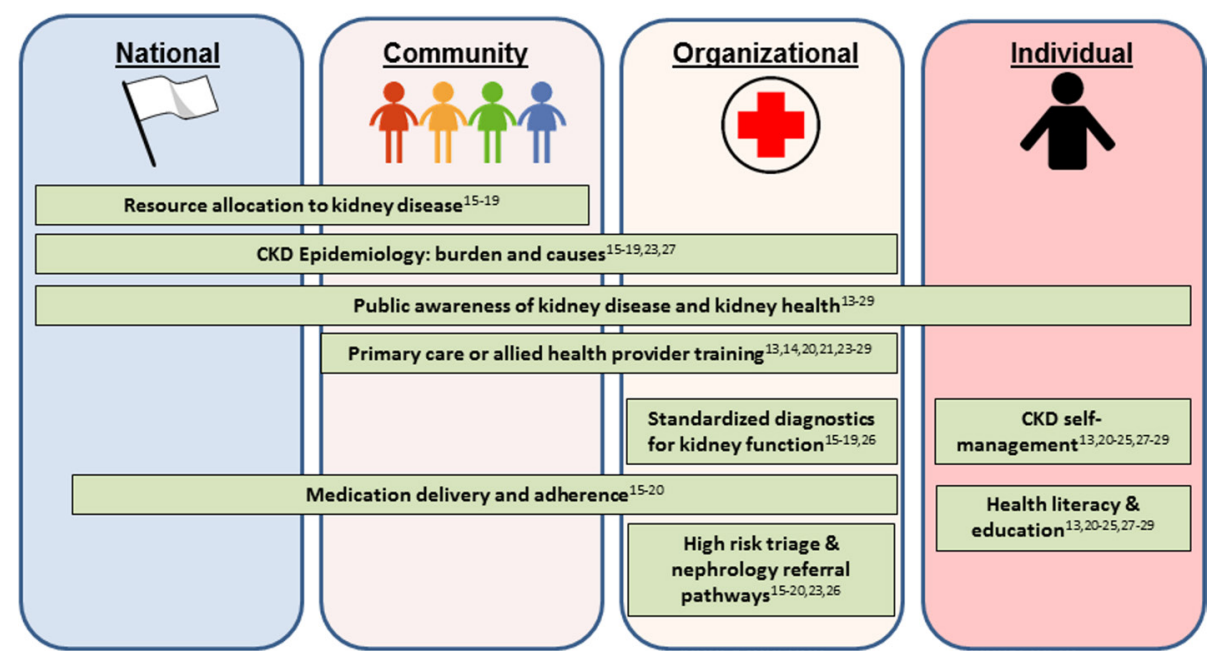

Figure 3 Challenges and opportunities for implementation of chronic kidney disease (CKD) care models in low- and middleincome countries (LMIC). 
After Taiwan identified aristolochic acid as an important contributing cause of CKD, the country launched massive education and regulation efforts to eliminate its use, a concerted effort which is at partly responsible for the declining rates of incident ESRD. ${ }^{35}$ Countries with high rates of CKD of unknown aetiology or populations with high frequencies of apolipoprotein-1 nephropathy risk alleles, for example, may need to consider unique pathways to screen for CKD. Furthermore, lifestyle and dietary changes portend a rise of chronic disease such as diabetes and hypertension, but at the same time, densely crowded cities with poor infrastructure, sanitation and waste disposal mean populations are also exposed to several infectious and environmental toxins. These populations are especially vulnerable, and public health strategies and CKD care models must reflect these broad risk factors. As more LMICs develop population-based epidemiological surveys to assess chronic disease burden, it will be critical to include an assessment of burden and cause of CKD in these efforts.

On a local and clinical level, a majority of the LMIC care models attempted to create a multidisciplinary clinic with variable levels of success reported. Many HICs have tested CKD care models incorporating components of the chronic care model,${ }^{8}$ which have shown cost-effective improvements in slowing progression of CKD. ${ }^{9}$ Longterm data from a randomised controlled trial evaluating the role of nurse care coordinators showed lower likelihood of a composite outcome of incident ESRD, death or $50 \%$ increase in serum creatinine. The CKD care models in LMICs spanned several approaches but in the traditional nephrologist-based clinics, personnel, medication and patient-level constraints were evident. ${ }^{14629}$

What seemed to be more successful was a focus on prevention through decision support for primary care or allied health professional training. The strongest example of this strategy was the cluster randomised trial equipping community healthcare workers and primary care providers to better manage hypertension, resulting in preservation of kidney function over the long term. ${ }^{13}$ The recent description of a successful multidisciplinary CKD clinic in Thailand also relied heavily on community healthcare workers to ensure frequent patient interactions and consistent medication use. ${ }^{20}$

More of these care models have been sustainable, and if extended to include referral pathways could represent a scalable approach to CKD care. ${ }^{1323} 27$ But even these models require rigorous effectiveness and cost-effectiveness data to gain traction among policymakers. For example, Mani et $a l \mathrm{~s}^{27}$ care model leveraged the community's structure and resources (employing young educated girls living in the serviced community as initial screeners and mobilising dedicated physicians for monthly in-community clinics), and on the basis of described costs, was affordable, but lacked rigorous ascertainment of the demographics of persons receiving the care, or outcomes in comparison with a similar receiving usual care.
We note several limitations to our study. We did not review non-indexed journals, which could introduce a slight publication bias, especially from LMICs where access to publication or indexing may be limited. In many instances, care models may be considered quality-improvement rather than original research, and we did not assess unpublished works. We did not report summary findings and our results are descriptive in nature, and caution must be applied when comparing studies from different settings and populations. Finally, despite corresponding with several authors from the included studies, we were unable to correspond with all authors, and follow-up data on the sustainability or effectiveness of some care models were not available.

In conclusion, few rigorous CKD care models have been reported from LMICs, and data are limited on the optimal methods for effective, affordable and sustainable CKD care. The most effective CKD care models integrated CKD care into national chronic disease programmes, or focused on bolstering primary care or allied health professional knowledge and services. To draw wider uptake, future work on CKD care models will need to address region-specific causes of CKD, increase highquality diagnostic capabilities, establish referral pathways and, most importantly, provide clear assessments of effectiveness and cost-effectiveness.

Acknowledgements We thank the authors of included manuscripts who provided follow-up on their care models.

Contributors JWS generated search strategy, undertook article review, coled manuscript writing and led figure generation. MVI undertook systematic search. GMC was involved in critical review of manuscript. SA was involved in conception of research, undertook article review and coled manuscript writing.

Funding JWS was supported by the American Kidney Fund Clinical Scientist in Nephrology Fellowship. SA was supported by the National Institute for Diabetes and Digestive and Kidney Health (grant number K23 DK101826).

\section{Competing interests None declared.}

Provenance and peer review Not commissioned; externally peer reviewed.

Data sharing statement Additional data are available upon request to the corresponding author.

Open Access This is an Open Access article distributed in accordance with the Creative Commons Attribution Non Commercial (CC BY-NC 4.0) license, which permits others to distribute, remix, adapt, build upon this work non-commercially, and license their derivative works on different terms, provided the original work is properly cited and the use is non-commercial. See: http://creativecommons.org/ licenses/by-nc/4.0/

(C) Article author(s) (or their employer(s) unless otherwise stated in the text of the article) 2018. All rights reserved. No commercial use is permitted unless otherwise expressly granted.

\section{REFERENCES}

1. Liyanage $T$, Ninomiya $T$, Jha $V$, et al. Worldwide access to treatment for end-stage kidney disease: a systematic review. Lancet 2015;385:1975-82.

2. Anand S, Bitton A, Gaziano T. The gap between estimated incidence of end-stage renal disease and use of therapy. PLoS One 2013;8:e72860.

3. Bello AK, Levin A, Tonelli M, et al. Assessment of Global Kidney Health Care Status. JAMA 2017;317:1864-81.

4. Jafar $\mathrm{TH}$, Stark $\mathrm{PC}$, Schmid $\mathrm{CH}$, et al. Progression of chronic kidney disease: the role of blood pressure control, proteinuria, and 
angiotensin-converting enzyme inhibition: a patient-level metaanalysis. Ann Intern Med 2003;139:244-52.

5. Gaede P, Lund-Andersen $\mathrm{H}$, Parving $\mathrm{HH}$, et al. Effect of a multifactorial intervention on mortality in type 2 diabetes. $N$ Engl $J$ Med 2008;358:580-91.

6. Rauen T, Eitner F, Fitzner C, et al. Intensive Supportive Care plus Immunosuppression in IgA Nephropathy. N Engl J Med 2015;373:2225-36.

7. Jungers $P$, Joly D, Barbey F, et al. ESRD caused by nephrolithiasis: prevalence, mechanisms, and prevention. Am J Kidney Dis 2004;44:799-805.

8. Quality AfHRa. Introduction to the Care Model. Practice Facilitation Handbook, 2013.

9. Johns TS, Yee J, Smith-Jules T, et al. Interdisciplinary care clinics in chronic kidney disease. BMC Nephrol 2015;16:161.

10. The World Bank. World Bank Country and Lending Groups, 2017.

11. Higgins JP, Altman DG, Gøtzsche PC, et al. The Cochrane Collaboration's tool for assessing risk of bias in randomised trials. BMJ 2011;343:d5928.

12. von Elm E, Altman DG, Egger M, et al. The Strengthening the Reporting of Observational Studies in Epidemiology (STROBE) statement: guidelines for reporting observational studies. J Clin Epidemiol 2008;61:344-9.

13. Jafar TH, Allen JC, Jehan I, et al. Health Education and General Practitioner Training in Hypertension Management: LongTerm Effects on Kidney Function. Clin J Am Soc Nephrol 2016;11:1044-53.

14. Garcia-Garcia G, Martinez-Castellanos Y, Renoirte-Lopez K, et al. Multidisciplinary care for poor patients with chronic kidney disease in Mexico. Kidney Int Suppl 2013;3:178-83.

15. Almaguer M, Herrera R, Alfonzo J, et al. Chronic kidney disease in Cuba: epidemiological studies, integral medical care, and strategies for prevention. Ren Fail 2006;28:671-6.

16. Almaguer M, Herrera R, Alfonso J, et al. Primary health care strategies for the prevention of end-stage renal disease in Cuba. Kidney Int 2005;68:S4-S10.

17. Hooi LS, Wong HS, Morad Z. Prevention of renal failure: the Malaysian experience. Kidney Int Suppl 2005;94:S70-S74.

18. Tapia-Conyer R, Gallardo-Rincón H, Saucedo-Martinez R. CASALUD: an innovative health-care system to control and prevent non-communicable diseases in Mexico. Perspect Public Health 2015;135:180-90.

19. Mastroianni-Kirsztajn G, Bastos MG, Burdmann EA. Strategies of the Brazilian chronic kidney disease prevention campaign (2003-2009). Nephron Clin Pract 2011;117:c259-c265.

20. Jiamjariyapon $T$, Ingsathit A, Pongpirul K, et al. Effectiveness of Integrated Care on Delaying Progression of stage 3-4 Chronic
Kidney Disease in Rural Communities of Thailand (ESCORT study): a cluster randomized controlled trial. BMC Nephrol 2017;18:83.

21. Tang Q, Yang B, Fan F, et al. Effects of individualized exercise program on physical function, psychological dimensions, and health-related quality of life in patients with chronic kidney disease: A randomized controlled trial in China. Int $J$ Nurs Pract 2017;23:e12519.

22. Cortés-Sanabria L, Cabrera-Pivaral CE, Cueto-Manzano AM, et al. Improving care of patients with diabetes and CKD: a pilot study for a cluster-randomized trial. Am J Kidney Dis 2008;51:777-88.

23. Katz IJ, Hoy WE, Kondalsamy-Chennakesavan S, et al. Chronic kidney disease management--what can we learn from South African and Australian efforts? Blood Purif 2006;24:115-22.

24. Barahimi $\mathrm{H}$, Zolfaghari $\mathrm{M}$, Abolhassani $\mathrm{F}$, et al. E-Learning Model in Chronic Kidney Disease Management: a Controlled Clinical Trial. Iran J Kidney Dis 2017;11:280-5.

25. Cueto-Manzano AM, Martínez-Ramírez HR, Cortés-Sanabria L. Comparison of primary health-care models in the management of chronic kidney disease. Kidney Int Suppl 2013;3:210-4.

26. Fogazzi GB, Attolou V, Kadiri S, et al. A nephrological program in Benin and Togo (West Africa). Kidney Int 2003;63:S56-S60.

27. Mani MK. Experience with a program for prevention of chronic renal failure in India. Kidney Int Suppl 2005;94:S75-S78.

28. Tungsanga K, Ratanakul C, Pooltavee W, et al. Experience with prevention programs in Thailand. Kidney Int Suppl 2005;94:S68-S69.

29. Zhang $\mathrm{AH}$, Zhong $\mathrm{H}$, Tang $\mathrm{W}$, et al. Establishing a renal management clinic in China: initiative, challenges, and opportunities. Int Urol Nephrol 2008;40:1053-8.

30. 22nd Report of the Malaysian Dialysis and Transplant Registry 2014. Kuala Lumpur: The National Renal Registry, 2015.

31. Hooi LS, Ong LM, Ahmad G, et al. A population-based study measuring the prevalence of chronic kidney disease among adults in West Malaysia. Kidney Int 2013;84:1034-40.

32. Anand S, Zheng Y, Montez-Rath ME, et al. Do attributes of persons with chronic kidney disease differ in low-income and middleincome countries compared with high-income countries? Evidence from population-based data in six countries. BMJ Glob Health 2017;2:e000453.

33. Stanifer JW, Muiru A, Jafar TH, et al. Chronic kidney disease in low- and middle-income countries. Nephrol Dial Transplant 2016;31:868-74.

34. Sakai T, Kitagawa T. Screening system for asymptomatic renal disease in children in Japan. Acta Paediatr Jpn 1990;32:677-81.

35. Hwang SJ, Tsai JC, Chen HC. Epidemiology, impact and preventive care of chronic kidney disease in Taiwan. Nephrology 2010;15(Suppl 2):3-9. 